\title{
Mössbauer, XRD and TEM Study on the Intercalation and the Release of Drugs in/from Layered Double Hydroxides
}

\author{
E. Kuzmann,,${ }^{1,2, *}$ V. K. Garg, ${ }^{2}$ A. C. de Oliveira, ${ }^{2}$ L. Herojit Singh, ${ }^{2}$ S. S. Pati, ${ }^{2}$ E. M. Guimaraes, ${ }^{3}$ \\ Tatiane O. dos Santos, ${ }^{4}$ M. Ádok-Sipiczki, ${ }^{5}$ P. Sipos, ${ }^{5}$ I. Pálinkó ${ }^{6}$
}

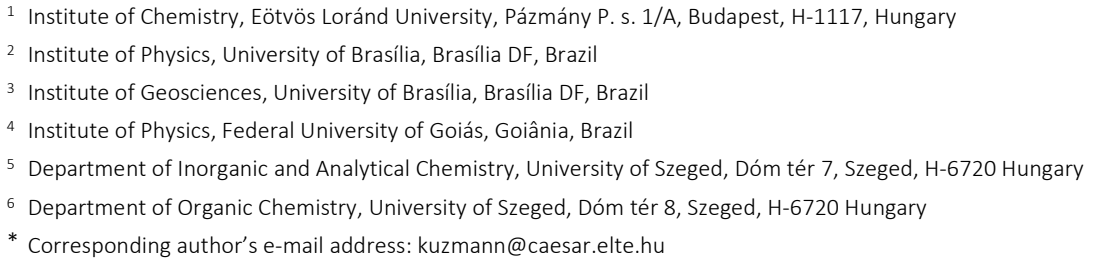

RECEIVED: June 15, 2015 * REVISED: September 25, 2015 * ACCEPTED: September 25, 2015

THIS PAPER IS DEDICATED TO DR. SVETOZAR MUSIĆ ON THE OCCASION OF HIS 70

Abstract: Layered double hydroxides (LDHs) are one of the very important nano-carriers for drug delivery, due to their many advantageous features, such as the ease and low-cost of preparation, low cytotoxicity, good biocompatibility, protection for the intercalated drugs, and the capacity to facilitate the uptake of the loaded drug in the cells. In our previous studies, Mössbauer spectroscopy was applied to monitor structural changes occurring during the incorporation of $\mathrm{Fe}(\mathrm{III})$ in $\mathrm{MgFe}$ - and $\mathrm{CaFe}-\mathrm{LDHs}$, and the intercalation of various organic compounds in anionic form. Recently, we have successfully elaborated a protocol for the intercalation and release of indol-2-carboxylate and L-cysteinate in CaFe-LDH. The corresponding structural changes in the LDH samples were studied by XRD, HR-TEM and ${ }^{57}$ Fe Mössbauer spectroscopy. The Mössbauer spectra reflected small but significant changes upon both the intercalation and the release of drugs. The changes in the basal distances could be followed by XRD measurements, and HR-TEM images made these changes visible.

Keywords: layered double hydroxides, Mössbauer spectroscopy, drug introduction, drug release, HR-TEM, XRD.

\section{INTRODUCTION}

$\mathbf{N}$ ANOMEDICINES have great potential to address some of the big problems in cancer therapy, such as how to get enough of the right drug to the right place without causing side effects or inducing drug resistance. Cellspecific targeting can be accomplished by attaching drugs to specially designed nanocarriers. ${ }^{[1]}$ Various moieties like iron oxide, gold, layered double hydroxide, calcium phosphate and silica nanoparticles, fullerenes and carbon nanotubes can be used as nano-carriers. Knowing the nature and properties of chemical bonds in the drug nano-carriers has high importance not only for advanced applications but from the point of view of toxicity, too.
Layered double hydroxides (LDHs) are among the very important nano-carriers for drug delivery, due to their many advantageous features, such as the ease and low-cost of preparation, low cytotoxicity, good biocompatibility, protection for the intercalated drugs, and the capacity to facilitate the uptake of the loaded drug in the cells. ${ }^{[2,3]}$

LDHs, also known as anionic clays, are found in $\mathrm{Na}$ ture and many of their representatives belong to the hydrotalcite supergroup. For use, they are mostly synthesised. The most often applied method is the co-precipitation of the component salts with the help of a base in solution. ${ }^{[4]}$

LDHs can be represented by the general formula of $\left[\mathrm{M}^{2+}{ }_{1-x} \mathrm{M}^{3+}{ }_{x}(\mathrm{OH})_{2}\left(\mathrm{~A}^{n-}\right)_{x / n}\right] \cdot y \mathrm{H}_{2} \mathrm{O}$, where $\mathrm{M}^{2+}$ is a divalent 


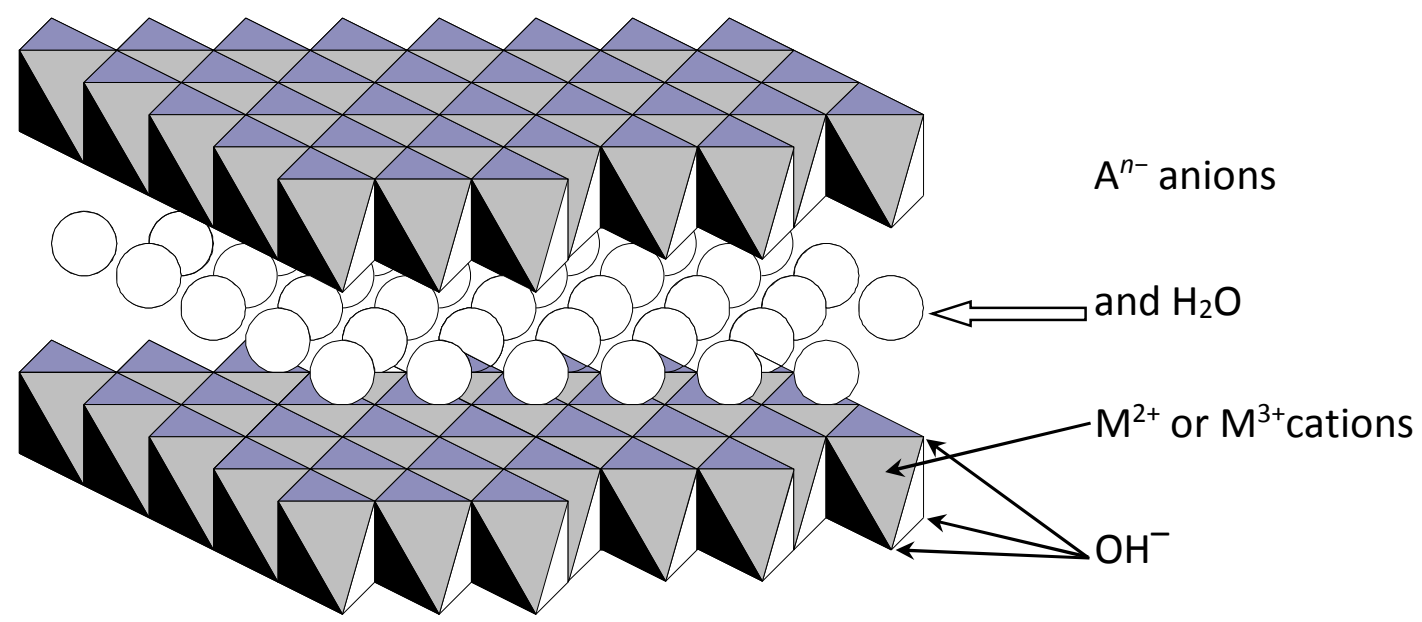

Figure 1. Schematic view of the LDH structure.

cation, $\mathrm{M}^{3+}$ is a trivalent cation, $\mathrm{A}^{n-}$ is an interlayer anion with charge $n$ and $x$ and $y$ are fraction constants. ${ }^{[5]}$ The metal cations occupy the centres of edge-shared octahedra, whose vertices contain hydroxide ions connected to form infinite two-dimensional sheets (Figure 1). Between the positively charged metal hydroxide layers, fully or partially hydrated simple inorganic anions are situated in order to compensate the positive charges of the layers. These anions can be exchanged to more complex organic anions forming organic-inorganic functional nanocomposites having technological importance in catalysis, ${ }^{[6]}$ nanocomposite materials engineering, ${ }^{[7]}$ medical and pharmaceutical sciences. ${ }^{[3]}$

A number of cardiovascular or anti-inflammatory agents, either carboxylic acids or carboxylic derivates could be ion-exchanged with LDHs to have controlled release. ${ }^{[8-11]}$ Other drugs have already been introduced between the hydroxide layers ${ }^{[3,12,13]}$ as well.

There are several ways for the intercalation of drugs into the interlayer space of LDH, like via anion exchange or including the drug anion in co-precipitating mixture or via calcination-rehydration using the "memory effect" of LDHs. ${ }^{[14]}$

${ }^{57} \mathrm{Fe}$ Mössbauer spectroscopy ${ }^{[15,16]}$ is a useful technique to characterise LDHs, in which $\mathrm{Fe}^{3+}$ ions are constituents of the layers. In our previous works on LDHs, ${ }^{[17-20]}$ Mössbauer spectroscopy revealed whether the iron was in the $\mathrm{LDH}$ structure formed or not ${ }^{[17]}$ when the $\mathrm{Ca}: \mathrm{Fe}^{[18]}$ or $\mathrm{Mg}: \mathrm{Fe}^{[19]}$ ratios were altered. It could also provide with structural information when different organic anions were intercalated into a CaFe LDH. ${ }^{[20]}$

In this paper, the syntheses of two functional nanocomposites are described using CaFe-LDH as nanocarrier and cysteinate or indole-2-carboxylate as anionic intercalated drugs. The effects of sodium carbonate on the release of drugs are examined and characterisation of the obtained substances is given by XRD, TEM and ${ }^{57} \mathrm{Fe}$ Mössbauer spectroscopy. The work was performed with the aim of preparing durable composites, which are able to act as controlled released drugs in pharmaceutical applications.

\section{EXPERIMENTAL}

\section{Chemicals and Sample Preparation}

All materials used for the syntheses of either the LDHs [calcium chloride $\left(\mathrm{CaCl}_{2}\right.$, Molar Chemicals, puriss), iron chloride $\left(\mathrm{FeCl}_{3} \cdot 6 \mathrm{H}_{2} \mathrm{O}\right.$, Molar Chemicals, puriss special), hydrogen chloride $(\mathrm{HCl})$, sodium hydroxide $(\mathrm{NaOH}, \mathrm{VWR}$, a.r. grade)] were used as received without further purification. The concentrations of the iron-containing solutions were determined iodometrically. Millipore Millia water was used throughout the experiments.

LDH synthesis was performed with co-precipitation method with $\mathrm{NaOH}$ solution using chloride salts. In a typical synthesis of $\mathrm{Ca}$ (II) / Fe(III)-LDH, $\mathrm{NaOH}$ solution ([NaOH] $]_{T}=3-$ $5 \mathrm{M}$ ) was added dropwise to the vigorously stirred and, in a set of experiments, $\mathrm{N}_{2}$-blanketed solution containing the salts of the divalent metal ions $\left(\mathrm{CaCl}_{2}\right)$ and the trivalent metal ions $\left(\mathrm{FeCl}_{3}\right)$. Hydrochloric acid $(\mathrm{HCl})$ was used to set the $\mathrm{pH}$ to $2.3 \mathrm{M} \mathrm{NaOH}$ solutions were used by the end of the syntheses when the $\left[\mathrm{OH}^{-}\right]$was set to $0.1 \mathrm{M}$. The molar ratio of divalent to trivalent metal was $2: 1$. The concentration of the iron solution was $0.1 \mathrm{M}$ and that of hydrochloric acid was $0.01 \mathrm{M}$. The resulting mixture was rapidly filtered until air dry in a practically $\mathrm{CO}_{2}$-free atmosphere, with the aid of a caustic resistant vacuum filter unit (Nalgene) equipped with an appropriate membrane (Versapor, 0.45 $\mu \mathrm{m})$. The solid material was washed with small amounts of pure and hot $\mathrm{NaOH}$ solution with the same concentration. 
The moisture sensitive crystals were kept in a desiccator over dry $\mathrm{SiO}_{2}$ at ambient temperature.

The preparation of drug-LDH nanocomposites were performed by the rehydration-dehydration method. The washed and dried LDH powder was hydrothermally treated in a Thermolyne 21100 tubular furnace for 6 hours at $T=$ $393 \mathrm{~K}$. The hydrothermally treated LDH powder was added to the aqueous ethanolic solutions (water: ethanol: $\mathrm{NaOH}$ $=5: 1: 1)$ of cysteine or indole-2-carboxylic acids, and the mixture was stirred for 7 days at $353 \mathrm{~K}$ under inert atmosphere. The resulting suspensions were filtered and washed three times, and then dried in a desiccator over dry $\mathrm{SiO}_{2}$.

To release the drugs from the $\mathrm{LDH}$, both the indol-2carboxylate- and the cysteinate-intercalated $\mathrm{Ca}_{2} \mathrm{Fe}-\mathrm{LDHs}$ were dissolved in $0.1 \mathrm{M} \mathrm{Na}_{2} \mathrm{CO}_{3}$ solution for 1 week. The exchange of anions occurred, since the $\mathrm{CO}_{3}{ }^{2-}$ ions are bonded much stronger between the layers of LDH than the drugs, and the total amount of the drug (indole-2-carboxylate or cysteinate) was released from the interlayer space. After the anion-exchange reaction, the product was filtered, washed and dried.

\section{Sample Characterisation Methods}

Powder X-ray diffraction (XRD) patterns of the air-dried and heat-treated solid samples were registered at room temperature in the $2 \Theta=3-70^{\circ}$ range by a Philips PW1710 instrument or by a Shimadzu 6000 diffractometer using CuKa $(\lambda=0.15418 \mathrm{~nm})$ radiation. Reflection positions were

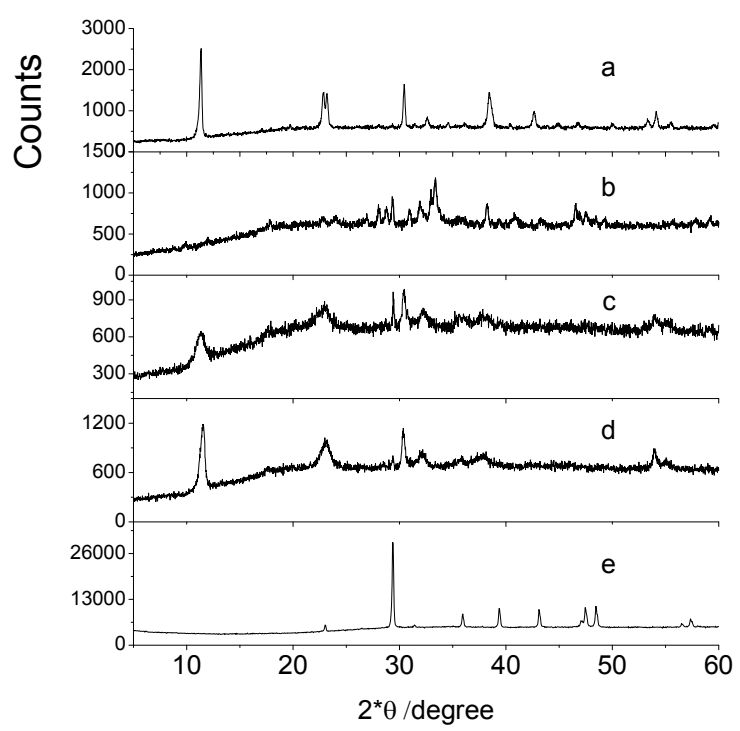

Figure 2. XRD patterns of pristine CaFe-LDH (a), heat-treated CaFe-LDH (b), cysteinate intercalated CaFe-LDH (c), indole2-carboxylate intercalated CaFe-LDH (d) and after releasing the intercalated cysteinate (e) or the intercalated indole-2carboxylate (f). determined via fitting a Gaussian function. They were found to be reproducible within $0.05^{\circ}$, therefore the uncertainty of the basal spacing was estimated to be $\pm 0.01 \mathrm{~nm}$. EXRAY code was used for the evaluation of diffractograms.

The samples were studied using a JEOL, JEM 2100 transmission electron microscope (TEM) at various magnifications (up to 1000000). Acceleration voltage was set to $200 \mathrm{kV}$. The samples were dissolved in ethanol before fixing them on a copper grid.

The ${ }^{57} \mathrm{Fe}$ Mössbauer spectra were recorded in transmission geometry using a commercial Wissel system operating in the constant acceleration mode. The radiation source used was the $50 \mathrm{mCi}{ }^{57} \mathrm{Co}$ in Rh-matrix. Samples (70 $\mathrm{mg}$ of powder) were placed in a sample holder with $1.6 \mathrm{~cm}$ in diameter. The Mössbauer spectrometer was calibrated with a thin natural iron sheet. The isomer shifts are given relative to $\alpha$-iron. Spectra were taken at 300 and $78 \mathrm{~K}$, the latter were recorded using a Janis cryostat. The Mössbauer spectra were evaluated by a least-square fitting of Lorentzians using the MOSSWINN code.

\section{RESULTS AND DISCUSSION}

XRD patterns of pristine and heat-treated CaFe-LDH, indole-2-carboxylate and cysteinate intercalated CaFe-LDHs and of those from which the drugs were released are shown in Figure 2.

The lattice parameters derived from the diffractograms are given in Table 1.

The XRD pattern of the pristine CaFe-LDH and its derived structural parameters (Table 1 ) are in agreement with those we used earlier ${ }^{[19]}$ showing that the initial LDH was prepared indeed by the applied co-precipitation method. Significant differences have been observed between the Xray diffractograms of heat-treated, intercalated and drugreleased CaFe-LDHs. The characteristic reflections of the LDH disappeared, while those of brownmillerite appeared in the XRD on heat treatment, reflecting the known phase transition due to the dehydration of the LDH materials. In the XRD patterns of both drug-intercalated compounds, the newly appeared characteristic diffraction lines of LDH structure show that the memory effect worked when the heattreated samples were placed under rehydrating conditions. This, together with the small but significant changes found in the lattice parameters and lattice spacing (Table 1) compared to those of pristine LDH indicate that the intercalation of drugs did occur. The $c$ values increased from 1.55 $\mathrm{nm}$ to $1.578 \mathrm{~nm}$ or decreased from $1.55 \mathrm{~nm}$ to $1.533 \mathrm{~nm}$ after cysteinate and indole-2-carboxylate intercalation into $\mathrm{CaFe}-\mathrm{LDH}$, respectively. The $a$ values remained almost constant at $\sim 0.587 \mathrm{~nm}$. This result revealed that the 2-dimensional lattice structures of the LDHs were neither decomposed nor dissolved during the intercalation. 
Table 1. Structural parameters of pristine, heat-treated, intercalated and drug-released CaFe-LDH.

\begin{tabular}{|c|c|c|c|c|c|}
\hline \multirow[t]{2}{*}{ Material } & \multirow[t]{2}{*}{$d_{003}(n m)$} & \multicolumn{3}{|c|}{ Lattice parameters (nm) } & \multirow[t]{2}{*}{ Assignment } \\
\hline & & $a$ & $b$ & $c$ & \\
\hline pristine CaFe-LDH & 0.778 & 0.58701 & & 1.5500 & CaFe-LDH \\
\hline heat-treated CaFe-LDH & & 0.5584 & 1.4600 & 0.5370 & brownmillerite \\
\hline cysteinate intercalated CaFe-LDH & 0.789 & 0.58703 & & 1.57779 & intercalated LDH \\
\hline indole-2-carboxylate intercalated CaFe-LDH & 0.767 & 0.58777 & & 1.53334 & intercalated LDH \\
\hline after release of intercalated cysteinate & & 0.49887 & & 1.70529 & calcium carbonate \\
\hline after release of intercalated indole-2-carboxylate & & 0.49887 & & 1.70529 & calcium carbonate \\
\hline
\end{tabular}

The basal spacings of the samples of cysteinate- and indole-2-carboxylate-intercalated CaFe-LDHs are different, due to the different charges, sizes and orientations of the gallery anions. Taking into account the $d_{003}$ of cysteinateintercalated LDH $(\sim 0.79 \mathrm{~nm})$ and the thickness of the LDH hydroxide basal layer $\left(0.18 \mathrm{~nm}^{[21]}\right)$, the gallery height is calculated to be $\sim 0.61 \mathrm{~nm}$, which is close to the length of the cysteinate anion $\left(0.56 \mathrm{~nm}^{[12]}\right)$. This suggests a possible orientation of interlayer cysteinate anions as shown in Figure 3 , where the anions are accommodated vertically in the interlayer region as a monolayer with the two negatively charged groups of individual anions attracted electrostatically to upper and lower hydroxide layers. This arrangement of cysteine anion corresponds to the one suggested for cysteinate intercalation into MgAl-LDH. ${ }^{[12]}$

A possible orientation of indole-2-carboxylate in the interlayer space of the CaFe-LDH can also be estimated from the comparison of the interlayer distance and the size of the organic anion. The gallery height can be calculated to be $\sim 0.58 \mathrm{~nm}$ (by substracting the thickness of the basal layer from the measured basal spacing). In the present case, however, considering the dimensions of the indole-2-carboxylate anion $\left(0.50 \times 0.30 \times 0.78 \mathrm{~nm}^{[20]}\right)$, there is not enough space to fit two horizontal rows of molecules in the interlayer of CaFe-LDH. A tilted vertical arrangement of a single row of anions may be a possible orientation as depicted in Figure 4, when the charge distribution can be somewhat similar to that occurring for the indole-2-carboxylate-intercalated CaFe-LDH prepared in aqueous acetone solution. ${ }^{[20]}$ This model is also supported by the Mössbauer results (Table 2 ).

The most striking result was found in the case of drug release from the LDH interlayers. All lines in the X-ray diffractograms were found to belong to the calcite type $\mathrm{CaCO}_{3}$, fully matching the fingerprint of the standard pattern (ASTM card No 83-0578). Consequently, the XRD pattern revealed that $\mathrm{CaCO}_{3}$ was the only crystalline phase in all the samples either the cysteinate or the indole-2-carboxylate anion was released from the CaFe-LDH induced by $\mathrm{Na}_{2} \mathrm{CO}_{3}$. The lack of the diffraction lines typical of the $\mathrm{LDH}$

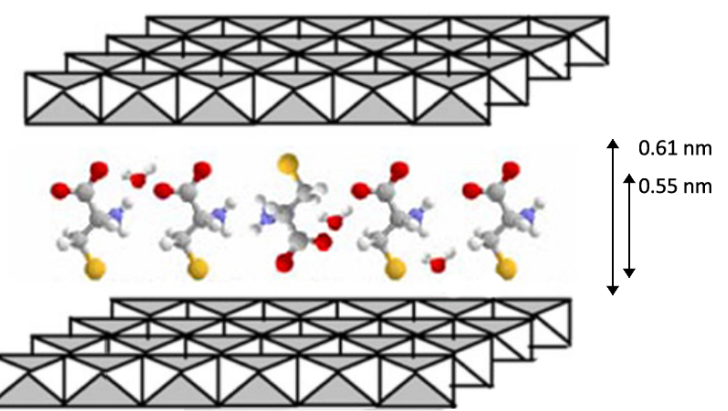

Figure 3. Schematic diagram for the possible orientation of the cysteinate ion in interlayer space of the CaFe-LDH (The arrows indicate the interlayer distance and the size of the anion).

and the appearance of $\mathrm{CaCO}_{3}$ evidence the decomposition of $\mathrm{LDH}$ structure during the drug release procedure. The release of intercalated drugs proceeded via the substitution of drug anions by $\mathrm{CO}_{3}{ }^{2-}$ anions. Since the $\mathrm{CO}_{3}{ }^{2-}$ ions bond much stronger between the layers of $\mathrm{LDH}$ than the organic

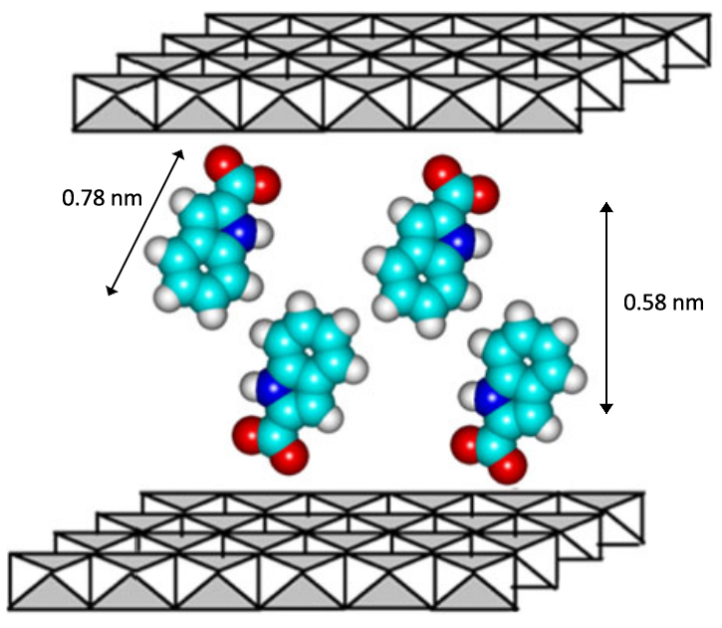

Figure 4. Schematic diagram for the possible orientation of indole-2-carboxylate in the interlayer space of CaFe-LDH. 
Table 2. Mössbauer parameters for the drug-intercalated/released CaFe-LDHs obtained from the spectra registered at $78 \mathrm{~K}$.

\begin{tabular}{ccc}
\hline Sample & $\delta(\mathrm{mm} / \mathrm{s}) / \mathrm{Fe}$ & $\Delta(\mathrm{mm} / \mathrm{s})$ \\
\hline pristine CaFe-LDH & $0.47 \pm 0.002$ & $0.46 \pm 0.004$ \\
$\mathrm{CaFe}-\mathrm{LDH}$ intercalated with indole-2-carboxylate & $0.47 \pm 0.002$ & $0.54 \pm 0.004$ \\
released ( with $\left.\mathrm{Na}_{2} \mathrm{CO}_{3}\right)$ from CaFe-LDH intercalated with indole-2-carboxylate & $0.47 \pm 0.002$ & $0.70 \pm 0.004$ \\
$\mathrm{CaFe}-\mathrm{LDH}$ intercalated with cysteinate & $0.47 \pm 0.002$ & $0.47 \pm 0.002$ \\
released $\left(\right.$ with $\left.\mathrm{Na}_{2} \mathrm{CO}_{3}\right)$ from CaFe-LDH intercalated with cysteinate & $0.70 \pm 0.004$ \\
\hline
\end{tabular}

anions, full exchange took place, and the total amount of the drug (indole-2-carboxylate or cysteinate) was released from the interlayer space. Under the applied circumstances, in $\mathrm{Na}_{2} \mathrm{CO}_{3}$ solution, the decomposition of LDH could occur according to the following reaction:

$$
2 \mathrm{Ca}(\mathrm{OH})_{2}+\mathrm{Na}_{2} \mathrm{CO}_{3}=\mathrm{CaCO}_{3}+2 \mathrm{NaOH}
$$

Our finding for the decomposition of LDH on the drug release is consistent with a recent work ${ }^{[22]}$ in which the dissolution of LDH was also observed on pyrophosphate removal by CaFe-LDH.

The above mentioned results were confirmed and complemented by Mössbauer spectroscopic measurements and the TEM results.

Typical Mössbauer spectra of pristine, intercalated indole-2-carboxylate and cysteinate-intercalated CaFe$\mathrm{LDHs}$ and of those from which the drugs were released are shown in Figures 5 and 6 . All spectra were satisfactorily decomposed into a doublet in accordance with the evaluation of Mössbauer spectra of other LDHs. ${ }^{[18-20]}$ The Mössbauer parameters are depicted in Table 2.

The Mössbauer spectrum and parameters of the pristine CaFe-LDH agrees well with those recorded earlier for the corresponding LDHs. ${ }^{[19,20]}$ This spectrum corresponds to high spin $\mathrm{Fe}^{3+}$ ions occupying the centres of shared octahedra, whose vertices contain hydroxide ions, which connect to form the layers of LDHs.

Significant and characteristic changes were found in the Mössbauer spectra (Figures 5 and 6) after the intercalation of drugs and after releasing the intercalated drugs.

The intercalation of both cysteinate and indole-2carboxylate into CaFe-LDH did not change the isomer shift compared to that of the pristine substance. This indicates that the electron density at the site of the iron nucleus remained the same. This local electron density is mainly determined by the hydroxides being in vicinity of iron inside the layer and it is not sensitive, at the resolution reflected by the Mössbauer isomer shift for high spin $\mathrm{Fe}^{3+}$, for the perturbation caused by the intercalation of different anions between the layers of CaFe-LDH. Similar results were obtained for different anions and preparation conditions in

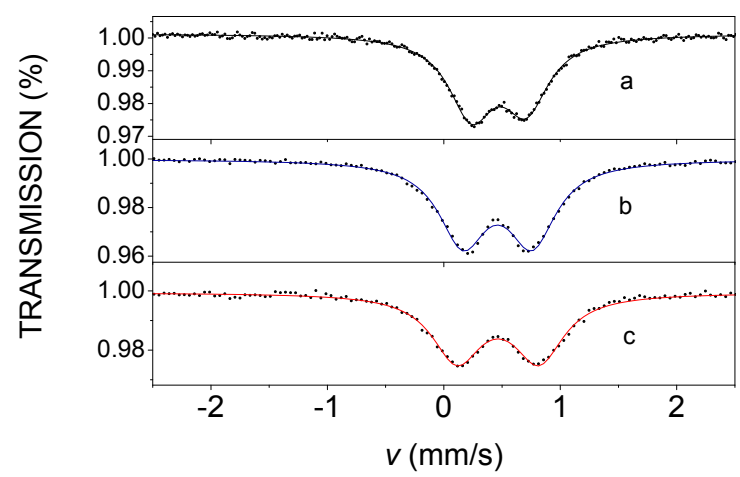

Figure 5. ${ }^{57} \mathrm{Fe}$ Mössbauer spectra of the pristine CaFe-LDH (a), the cysteinate-intercalated CaFe-LDH (b) and after releasing of intercalated cysteinate (c), registered at $78 \mathrm{~K}$.

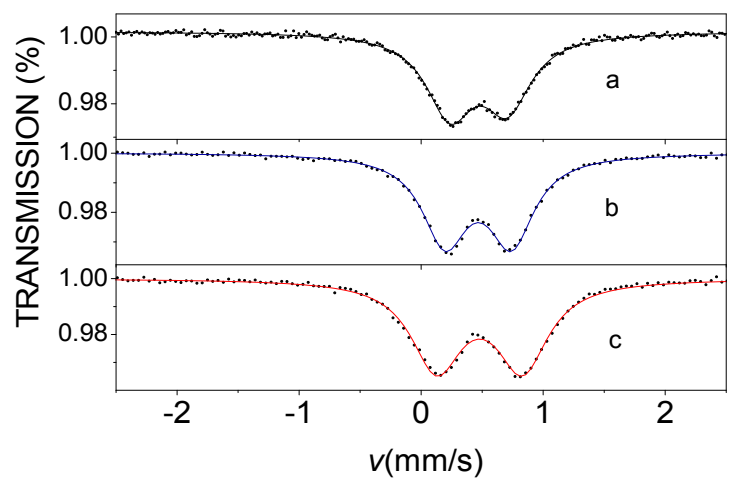

Figure 6. ${ }^{57} \mathrm{Fe}$ Mössbauer spectra of the pristine CaFe-LDH (a), the indole-2-carboxylate intercalated CaFe-LDH (b) and after releasing of intercalated indole-2-carboxylate (c), registered at $78 \mathrm{~K}$.

our previous work, ${ }^{[20]}$ too. The change in the isomer shift values recorded at $293 \mathrm{~K}$ and $78 \mathrm{~K}$ reflects only the corresponding regular temperature shift, ${ }^{[15]}$ in agreement with the characteristics of CaFe-LDH materials. ${ }^{[19]}$

However, the Mössbauer spectra of intercalated compounds registered either at $78 \mathrm{~K}$ (Table 2) or at $293 \mathrm{~K}$ showed significant differences in the quadrupole splitting compared to those of the pristine substance. 
An increase in the quadrupole splitting from 0.46 $\mathrm{mm} / \mathrm{s}$ to $0.54 \mathrm{~mm} / \mathrm{s}$ or to $0.59 \mathrm{~mm} / \mathrm{s}$ was observed (Table 2) upon the intercalation of indole-2-carboxylate and cysteinate, respectively. No considerable temperature dependence was detected in the quadrupole splitting between $293 \mathrm{~K}$ and $78 \mathrm{~K}$. These observed differences in quadrupole splitting values upon the drug intercalation into the LDHs are in the similar range to those obtained for another anion intercalation ${ }^{[20]}$ or when the $\mathrm{Ca}$ :Fe ratio was changed in LDHs. ${ }^{[19]}$

Since the quadrupole splitting is proportional with the local electric field gradient (EFG), the changes in quadrupole splitting can be connected with the changes in the EFG. The changes in the EFG are due to changes in the charge distribution around the iron atom, which can be caused by change in the molecule symmetry (spatial arrangement of ligands) or by the change in the molecular composition (by changing the charge of the ligand). ${ }^{[15]}$ In the case of the intercalation of the drugs into the CaFe-LDH, the increase in quadrupole splitting can be explained by lowering the symmetry of charge distribution in the compound. Furthermore, the intercalation of different anions into the interlayer space of the CaFe-LDH can modify the charge distribution differently, producing different ligand contributions to the EFG, consequently, resulting in different quadrupole splitting values. Therefore, the quadrupole splitting characteristic of an anion intercalated at a given condition can indicate the spatial arrangement of anions between the layer, as was demonstrated previously when the same anion was used but the solvent was different. ${ }^{[20]}$ The quadrupole splitting value $(0.54 \mathrm{~mm} / \mathrm{s})$ belonging to the CaFe-LDH intercalated with indole-2carboxylate under the present conditions differed significantly from that $(0.64 \mathrm{~mm} / \mathrm{s})$ reported earlier ${ }^{[20]}$ for the same anion intercalated also in aqueous ethanol solution. Furthermore, the presently found quadrupole splitting is in agreement with that observed previously upon the intercalation of indole-2-carboxylate in aqueous acetone solution, ${ }^{[20]}$ which suggests that the spatial arrangement of the anions between the layers can be close to that established upon intercalation with aqueous acetone solution. Since the XRD results excluded the spatial arrangement of anions, which was assigned for the aqueous ethanol solution ${ }^{[20]}$ and limited the original arrangement found at the intercalation with aqueous acetone solution, an arrangement with somewhat tilted anions is proposed as depicted in Figure 4.

The present Mössbauer results confirm the intercalation of both the indole-2-carboxylate and the cysteinate ions into CaFe-LDHs, and support the model proposed for the spatial arrangement of the anions between the layers (Figures 3 and 4).
Upon treatment with $\mathrm{Na}_{2} \mathrm{CO}_{3}$ solution to release the intercalated drugs from both the cysteinate- and indole-2carboxylate-intercalated CaFe-LDH, the Mössbauer spectra revealed a broader doublet with isomer shift $\delta=0.47 \mathrm{~mm} / \mathrm{s}$ and quadrupole splitting of $\delta=0.70 \mathrm{~mm} / \mathrm{s}$ at $78 \mathrm{~K}$. It was observed that typically the same Mössbauer parameters occurred at the release of both drugs from CaFe-LDHs both at $78 \mathrm{~K}$ and correspondingly at $293 \mathrm{~K}$, too. To assign this component, $\mathrm{CaCO}_{3}$ formation was taken into consideration, which occurred during the release reaction when the structure of the $\mathrm{LDH}$ decomposed and $\mathrm{CaCO}_{3}$ remained the only crystalline phase in the system according to the results of XRD and TEM analyses. Consequently, the doublet can be assigned to ferrihydrite, which is poorly ordered, often called "amorphous iron hydroxide" or hydrous iron oxide, which commonly forms in iron-containing solutions, and more importantly, its Mössbauer parameters match with those of ferrihydrite both at $78 \mathrm{~K}$ and at $293 \mathrm{~K} .{ }^{[23]}$ Note that another known iron-hydroxide or oxyhydroxide phases should give observable characteristic pattern contribution in the XRD of these samples, which was not the case.

Accordingly to the XRD, TEM (see later) and Mössbauer results obtained with the LDHs from which the intercalated drugs were released, the mechanism of the release process occurring in the $\mathrm{Na}_{2} \mathrm{CO}_{3}+$ drug-intercalated CaFeLDH solution, can be summarised as follows:

- Exchange of anions occurs, the $\mathrm{CO}_{3}{ }^{2-}$ ions effuse the drug from the interlayers of LDH

- The drug released from the LDH being in the solution, is filtered and washed out

- The structure of LDH gradually decomposed

- $\mathrm{CaCO}_{3}$ is formed

○ Ferrihydrite is formed

Our procedure for the drug release from LDH may be useful drug delivery method, since the LDH structure will be dissolved during the drug release and the formed components ( $\mathrm{CaCO}_{3}$ and ferrihydrite) are not toxic and digestible.

TEM micrographs of the pristine, the indole-2-carboxylate- and cysteinate-intercalated CaFe-LDHs and of those from which the drugs were released are shown in Figures 7-10. The HR-TEM results confirm the results obtained by XRD and Mössbauer spectroscopy.

HR-TEM micrographs of the pristine CaFe-LDH (Figure 7) reveal highly ordered structure showing the stacking of the LDH layers. The basal spacing value estimated from the TEM images is comparable to the one obtained by XRD analysis.

HR-TEM micrographs in Figures 7 and 8 show changes in the structure morphology compared to that of 

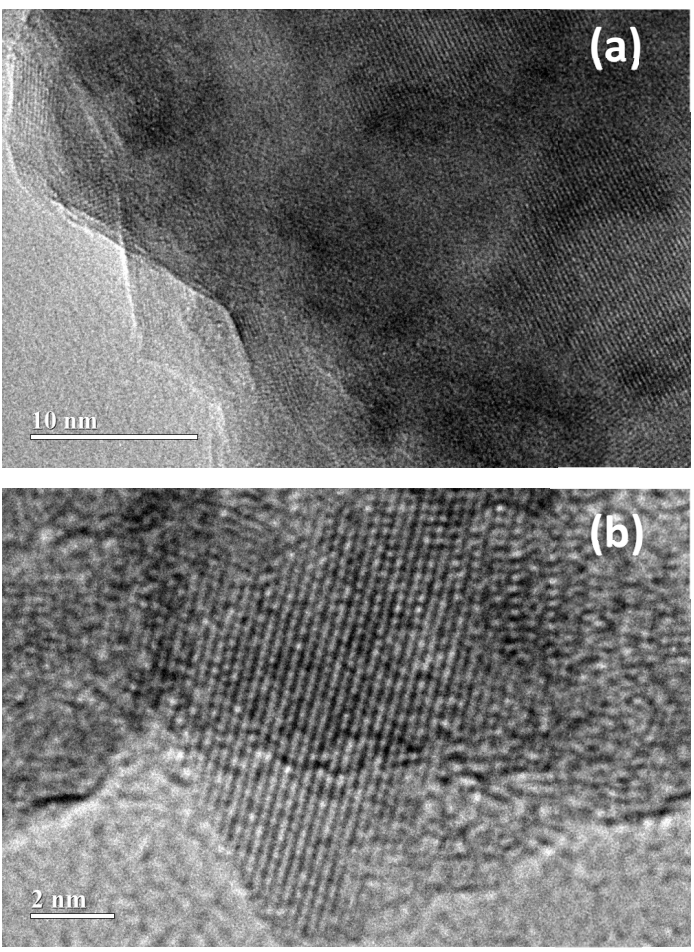

Figure 7. HR-TEM micrographs of pristine CaFe-LDH. Micrograph (b) was recorded at higher resolution than (a), as indicated by the scale bars.
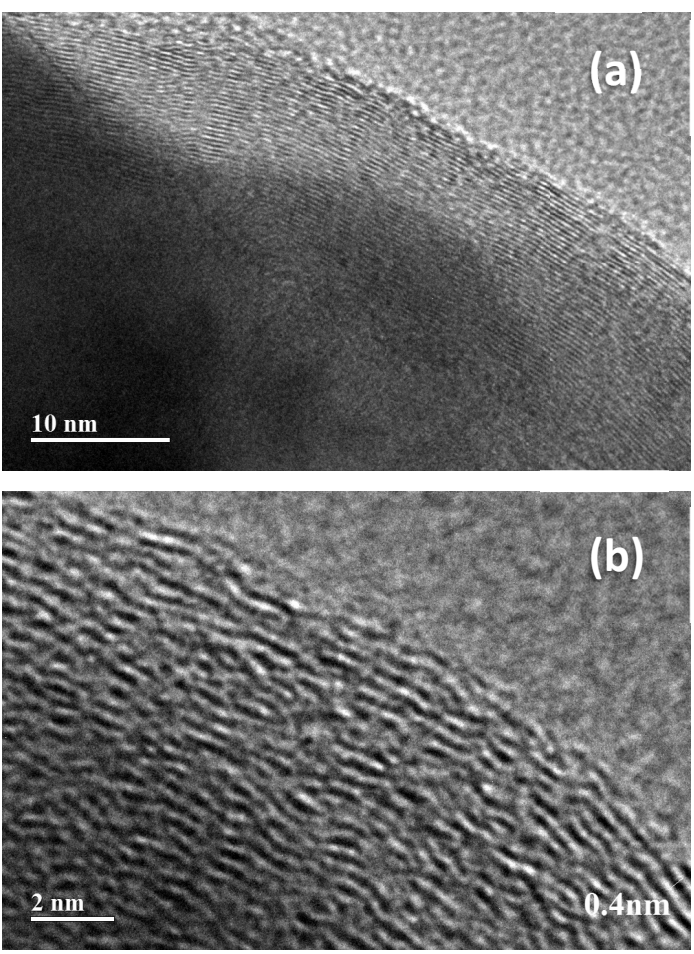

Figure 8. HR-TEM micrographs of the CaFe-LDH intercalated with indole-2-carboxylate. Micrograph (b) was recorded at higher resolution than (a), as indicated by the scale bars.
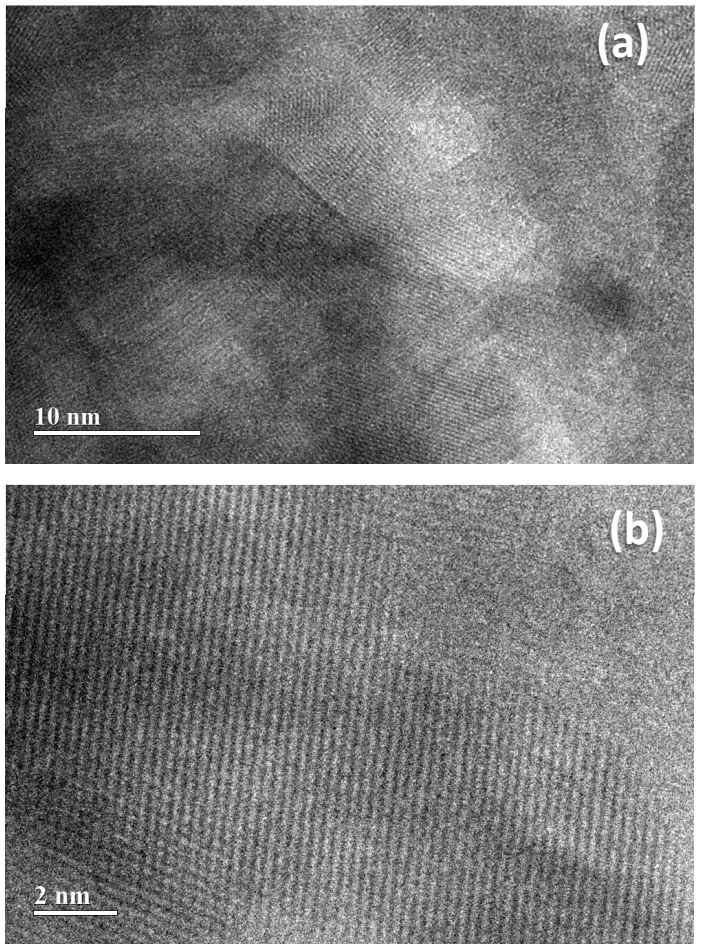

Figure 9. HR-TEM micrographs of CaFe-LDH intercalated with cysteinate anion. Micrograph (b) was recorded at higher resolution than (a), as indicated by the scale bars.
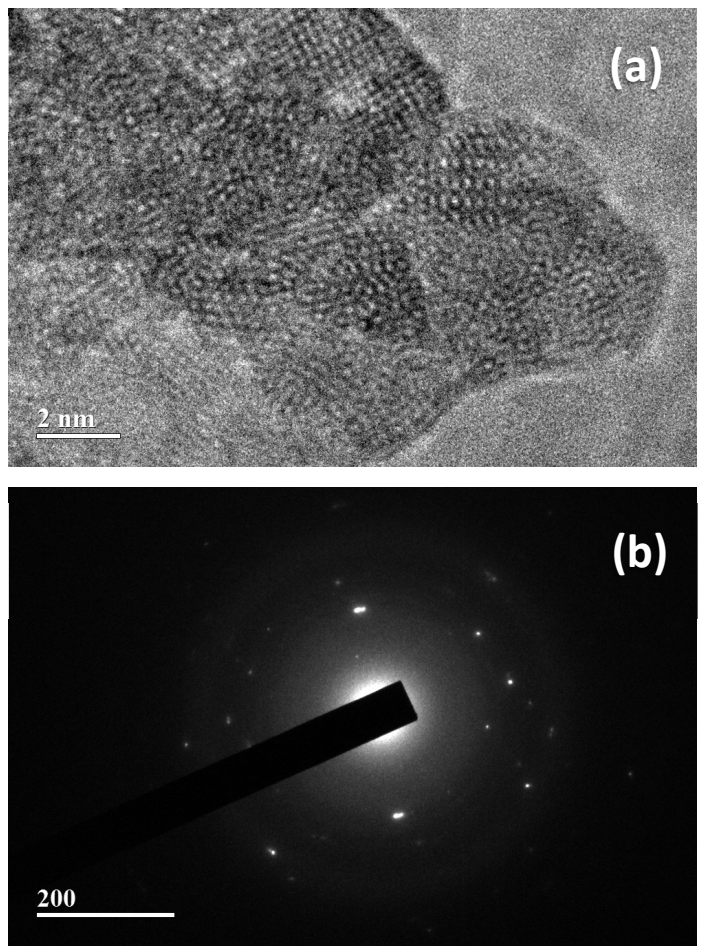

Figure 10. HR-TEM micrograph (a) and diffraction pattern (b) for the cysteinate-intercalated CaFe-LDH after release on $\mathrm{Na}_{2} \mathrm{CO}_{3}$. 
the pristine material, which is due to intercalation. The layered structure of LDH is preserved in both the cysteinateand indole-2-carboxylate-intercaleted LDHs, in good agreement with the XRD results. While the equidistance character of layers remains similar to that of the pristine compound after cysteinate intercalation (Figure 8), a fluctuation of intercalated layers are reflected for indole-2-carboxylate intercalation (Figure 7). This is consistent with the suggested models of orientation of intercalated anions in the LDH depicted in Figures 3 and 4, since the cysteinate anion can be accommodated easily between the layers, while indole-2-carboxylate can be hardly fitted, and it may cause some corrugation in the layers.

The morphology of intercalated CaFe-LDH after release with $\mathrm{Na}_{2} \mathrm{CO}_{3}$ (Figure 10) is significantly different from those shown in Figures 7-9. The layered structure changed to a granular one. The image is consistent with that of a precipitated calcite, and the electron diffraction pattern can also be associated with $\mathrm{CaCO}_{3},{ }^{[24]}$ since the value of $\mathrm{d}$-spacing correlates with those of calcite. Consequently, the TEM results can confirm the XRD result, i.e. the formation of $\mathrm{CaCO}_{3}$ occurs via the decomposition of $\mathrm{LDH}$ when the drug is released with $\mathrm{Na}_{2} \mathrm{CO}_{3}$.

\section{CONCLUSION}

Cysteinate and indol-2-carboxylate drug anions could be successfully introduced in-between the layers of CaFe-LDH. The success of intercalation was verified by XRD, HR-TEM and ${ }^{57} \mathrm{Fe}$ Mössbauer spectroscopic methods. Approximate views on the arrangement of anions between the layers were given taking into consideration the interlayer distances (XRD and TEM), size data (molecular modelling) and charge distribution (Mössbauer) of the anions.

Most importantly, it was shown that decomposition of $\mathrm{LDH}$ occurs at the release of drugs in $\mathrm{Na}_{2} \mathrm{CO}_{3}$ solution when $\mathrm{CaCO}_{3}$ and ferrihydrite are formed.

${ }^{57} \mathrm{Fe}$ Mössbauer spectroscopy is a suitable and useful method to characterise Fe-containing LDH materials.

Acknowledgement. The financial supports from the CAPESBrazil (No A127/2013) and OTKA NKFI106234 grants are thankfully acknowledged.

\section{REFERENCES}

[1] A. H. Faraji, P. Wippf, Bioorg. Med. Chem. 2009, 17, 2950.

[2] P. Nalawade, D. Nawar, J. Scientific and Industrial Res. 2009, 68, 268.

[3] X. Bi, H. Zhang, L. Dou, Pharmaceutics 2014, 6, 298.

[4] X. Duan, J. Lu, G. D. Evans, Assembly chemistry of anion-intercalated layered materials. In Modern
Synthetic Inorganic Chemistry (Eds.: Xu R., Pang W., Huo Q.), Elsevier B.V., Amsterdam, 2011, pp. 375404.

[5] F. Cavani, F. Trifirò, A. Vaccari, Catal. Today 1991, 11, 173.

[6] H. Shi, J. He, J. Catal. 2011, 279, 155.

[7] I. Pálinkó, Nanopages 2006, 1, 295.

[8] V Ambrogi, G. Fardella, G. Grandolini, P. Periolli, Int. J. Pharm. 2001, 220, 23.

[9] J. Yang, Y. Han, M. Park, T. Park, S. Hwang, J. Choy, Chem. Mater. 2007, 19, 2679.

[10] M. Z. Hussein, C. W. Long, Mater. Chem. Phys. 2004, 85, 427.

[11] S. Li, Y. Shen, M. Xiao, D. Liu, L. Fan, Z. Zhang, J. Nanomater. 2014, 862491

[12] M. Wei A. Guo, Z. Shi, Q. Yuan, M. Pu, G. Rao, X. Duan, J. Mater. Sci. 2007, 42, 2684.

[13] S. Choi, J. Choy, Nanomedicine 2011, 6(5), 803.

[14] Q. Wang, D. O'Hare, Chem. Rev. 2012, 112, 4124.

[15] P. Gütlich, E. Bill, A. Trautwein, Mössbauer Spectroscopy and Transition Metal Chemistry, SpringerVerlag GmbH, Berlin, Heidelberg, N.Y., 2011.

[16] E. Kuzmann, S. Nagy, A. Vértes, T. Weiszburg, V. K. Garg, Geological and mineralogical applications of Mössbauer spectroscopy In Nuclear Methods in Mineralogy and Geology, Techniques and Applications (Eds.: Vértes, A., Nagy, S., Süvegh, K.), Plenum Press, N.Y., 1998, pp. 285-376.

[17] D. Srankó A. Pallagi E. Kuzmann S. E. Canton M. Walczak A. Sápi, A. Kukovecz, Z. Kónya, P. Sipos, I. Pálinkó, Appl. Clay Sci. 2010, 48, 214.

[18] M. Sipiczki, E. Kuzmann Z. Homonnay, J. Megyeri, K. Kovács, I. Pálinkó, P. Sipos, Hyperfine Interactions 2013, 217, 145.

[19] M. Sipiczki, E. Kuzmann, Z. Homonnay, J. Megyeri K. Kovács, I. Pálinkó, P. Sipos, J. Mol. Struct. 2013, 1044, 116.

[20] M. Sipiczki, E. Kuzmann, Z. Homonnay, J. Megyeri, K. Kovács, I. Pálinkó, P. Sipos, Hyperfine Interactions 2014, 226. 171.

[21] I. Rousselot, C. Taviot-Guého, F. Leroux, P. Léone, P. Palvadeu, J.-P. Besse, J. Solid State Chem. 2002, 167, 137.

[22] Y. Wu, Y. Yu, J. Zhou, J. Liu, Y. Chi, Z. P. Xu, G. Qian, Chem. Eng. J. (Amsterdam, Neth.) 2012, 179, 72.

[23] E. Murad, J. H. Johnston, Iron Oxydes and Oxyhydroxides in Mössbauer Spectroscopy Applied to Inorganic Chemistry Vol. 2 (Ed.: G. J. Long), Plenum New York, London, 1987, p. 543.

[24] R. van de Locht, On The Nanostructure of Biogenic and Bio-Inspired Calcium Carbonate, as studied by electron microscopy techniques, PhD Dissertation, University of York, 2014. 